\title{
Cytoid, colloid and large intra-blister round bodies and c-ANCAs in a case of erythema multiforme
}

\author{
Ana Maria Abreu Velez¹, Vickie M. Brown², Michael S. Howard ${ }^{1}$ \\ ${ }^{1}$ Department of Georgia Dermatopathology Associates, Atlanta, Georgia, USA, ${ }^{2}$ Department of Family Dermatology, \\ Milledgeville, Georgia, USA
}

Corresponding author: Ana Maria Abreu Velez, MD PhD, E-mail: abreuvelez@yahoo.com

\begin{abstract}
Erythema multiforme is an acute, often self-limited and occasionally lethal syndrome with distinctive skin lesions and/or mucosal lesions; the disease has multiple triggers, including medications. We describe a 68 year old African American female on multiple medications; the patient suddenly presented with recurrent, flaccid bullae and erythematous circular lesions on her face, neck, genitals, hands and legs. Biopsies for hematoxylin and eosin (H\&E) examination, direct immunofluorescence (DIF) and immunohistochemistry (IHC) stains were performed. The histology displayed erythema multiforme with epidermal blisters, defragmented melanocytes and other cell debris inside the blister. DIF revealed deposits of IgG, IgM, IgA, IgD, Complement/C3, C4, and fibrinogen within the epidermis; and around upper dermal vessels. Anti-neutrophil cytoplasmic antibodies (c-ANCAS) were present as part of "large round bodies" inside the blisters, and as part of both cytoid and colloid bodies. Staining for Von Willembrand factor, CD68, myeloperoxidase and CD45 was seen on cell fragments within the blisters, as well as within the inflammatory infiltrate in the dermis. In this case cystoid, colloid and "large round bodies" may represent amalgamations of multiple cell fragments, with immunoglobulins and complement. Overall, a strong immunologic response was observed with c-ANCAs, caused by multiple medications.
\end{abstract}

Key words: Erythema multiforme; c-ANCAS; Cytoid and Colloid bodies

\section{INTRODUCTION}

Erythema multiforme (EM) represents an uncommon, acute inflammatory, reactive mucocutaneous disorder [1-3]. Etiologic agents of EM include infections (particularly herpes simplex virus, mycoplasma pneumonia, Epstein-Barr virus and histoplasmosis) and medications [1-3]. EM direct immunofluorescent (DIF) has been described as nonspecific, but may show deposition of Complement/C3 and fibrin along the dermal/epidermal junction, as well as $\operatorname{IgM}$, Complement/C3 and fibrin around upper dermal blood vessels [1-3]. Here we report an EM case that seemed to be triggered by multiple medications; the histologic and immunologic studies demonstrated a complex immune response, including anti-neutrophil cytoplasmic antibodies (c-ANCAs). We also noted cellular debris within the blisters, including fragments of melanocytes and keratinocytes.

\section{CASE REPORT}

A 68 year old African American female presented with recurrent, flaccid bullae; erosions, crusts, scales, hyperpigmented areas and erythematous circular lesions on her face, ears, neck, eyelids, genital areas, and hands and legs. The patient was taking multiple medications simultaneously, including Lasix ${ }^{\circledR}$, Omeprazol $^{\circledR}$, Omega 3, spironolactone, Norvasc ${ }^{\circledR}$, gentamicin, hydroxyzine, atenolol, Lisinopril ${ }^{\circledR}$, Zoloft $^{\circledR}$, eye drops, clonidine, Tylenol ${ }^{\circledR}$, Bumex, Procrit $^{\circledR}$, levothyroxine, and minoxidil. Biopsies for hematoxylin and eosin (H\&E) examination, DIF and

\footnotetext{
How to cite this article: Abreu Velez AM, Brown VM, Howard MS. Cytoid, colloid and large intra-blister round bodies and c-ANCAs in a case of erythema multiforme. Our Dermatol Online. 2017;8(3):282-285.

Submission: 26.11.2016; Acceptance: 17.02.2017

DOI: 10.7241 /ourd.20173.81
} 
immunohistochemistry (IHC) analysis were performed. A diagnosis of EM was established. The physician then began slowly removing each medication, trying to determine which ones could be triggering the disease; however, she was unable to determine which one(s) represented the exact triggering factor(s). The patient was prescribed Lidex ${ }^{\circledR}$ cream at $0.5 \%$; Tacrolimus ${ }^{\circledR}$ at $0.1 \%$; prednisone, 50 milligrams a day for two weeks, with a slow weekly decrease every week; and Allegra ${ }^{\circledR}$ at 180 milligrams daily. After three weeks of treatment, the rash cleared.

Our H\&E tissue processing and staining were performed as previously described $[4,5]$. Our case report was exempted from Institutional Review Board review and research patient consent.

\section{Microscopic Description}

The H\&E demonstrated a brisk, interface infiltrate of lymphocytes and histiocytes along the dermal/epidermal junction. Numerous necrotic keratinocytes were present within the epidermis, and vacuolation of basilar keratinocytes was also observed (Fig. 1). Intraepidermal and subepidermal blisters were present, and focal necrosis of one blister roof was seen. Lymphocytes, histiocytes, neutrophils and defragmented melanocytes were noted within the blister lumens as well as around

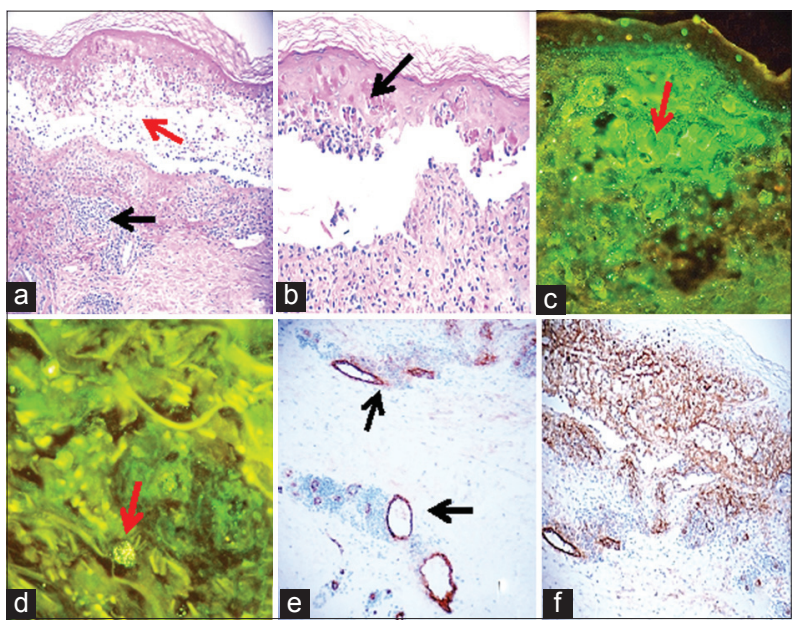

Figure 1: (a) H\&E staining showing a large subepidermal blister with cellular debris, lymphocytes, histiocytes and melanocytes within the blister lumen (red arrow), and a significant infiltrate featuring many of the same cells around upper dermal vessels (black arrow) (200X). (b) Colloidal bodies at likely sites of keratinocyte necrosis are noted in the epidermis (red arrow), (400X). (c) DIF, showing positive staining on colloid bodies in the epidermis using FITC conjugated anti-human fibrinogen (yellow staining; red arrow) (400X). (d) DIF, highlighting c-ANCAs positive in the dermis using FITC conjugated anti-human IgM antibody (red arrows). (e and f) IHC positive for von Willembrand factor, in e on dilated dermal vessels (black arrows)(400x), and inside a subepidermal blister in $\mathrm{f}$ within blister "debris" (200X). the upper dermal blood vessels, and some eccrine glands. Focal leukocytoclastic vasculitis was noted. The upper dermal blood vessels were dilated and showed compartmentalization and tropism toward the epidermal and subepidermal blisters. The PAS staining was positive on the cytoid, colloidal and "large round bodies".

\section{DIF}

Displayed IgG $(++$, focal epidermal (c-ANCAs); $\operatorname{IgA}(++$, on intra-epidermal "large round bodies", c-ANCAs, and epidermal and dermal cell junctions); $\operatorname{IgM}(++$, focal inside the blister intra-epidermal "large round bodies" and c-ANCAs); IgM $(++$, cytoid epidermal bodies), $\operatorname{IgD}(-)$; $\operatorname{IgE}(++$, focal epidermal c-ANCAs); Complement/Clq (++, focal intra-epidermal "large round bodies"), focal basement membrane zone (BMZ) staining; and strong $(++++)$ epidermal, and dermal and epidermal cell junctions and vessels; Complement/C3 $(++$, focal intra-epidermal "large round bodies", perivascular, perieccrine and dermal and epidermal cell junctions), Complement/C4 $(++$, intra-epidermal "large round bodies", and subcorneal c-ANCAs), Kappa light chains $(++$, focal intra-epidermal "large round bodies", and c-ANCAs); Lambda light chains (++, focal intra-epidermal "large round bodies" and c-ANCAs); albumin $(+$, focal dermal perivascular) and fibrinogen $(++++$, on "large round bodies" and vessels, linear focal BMZ, and dermal and epidermal cell junctions) (Fig. 1)

\section{IHC results}

HLA-DP,DQ,DR antigen was weakly positive on cells around the upper dermal blood vessels, however, in contrast to most autoimmune skin diseases, the vessels themselves were not clearly positive. Dilatation of dermal vessels (including lymphatics) was noted with von Willembrand factor and D2-40/podoplanin. CD68 positive cells were found in the blisters, mostly fragmented; and, also as part of the dermal infiltrate. Von Willembrand factor staining was seen inside the epidermal blister (in fragments), and overexpressed on many dermal vessels that showed epidermal trophism. $\operatorname{IgA}$ and $\operatorname{IgD}$ were positive on dermal blood vessels stained with von Willembrand factor. Factor XIIIa was positive around the dermal vessels; CD3, CD 8 and CD45 positive cells were seen as part of the dermal perivascular inflammatory infiltrate. Myeloperoxidase staining was positive on fragmented cells inside the blisters, and around the dermal vessels. The dermal 
vessels also stained positive for Complement/Clq, and fibrinogen.

\section{DISCUSSION}

The immune response in situ in EM has been studied, but remains incompletely characterized [1-3]. One group of authors studied several cases of $\mathrm{EM}$, using $\mathrm{H} \& \mathrm{E}$ and DIF and reported positivity in $88 \%$ of forty-one skin biopsy specimens from thirty-four patients, for the presence of cytoid bodies, or fluorescent keratinocytes in the epidermis [6]. These authors noted that the cytoid bodies stained most often with IgM (homogeneously) or with Complement/C3 (speckled) [6]. Our findings are in agreement with them, as well as with other authors who tested colloid bodies: IgG, IgA, IgM, C3 and fibrinogen could be demonstrated by DIF [7]. We noted both colloid bodies and cytoid bodies using H\&E (both represent eosinophilic hyaline ovoid bodies which are often found in the subepidermal papillary regions, or sometimes in the epidermis); and by DIF, primarily in the epidermis.

In addition, we observed some "large round bodies" exclusively inside the blisters that were much larger than cytoid bodies and/or colloid bodies. To our knowledge these "large round bodies" have never been previously described, and we speculate that these may be indicative of the fact that our patient was taking so many medications, possibly causing more significant tissue damage. Using double color IHC staining and multiple fluorochome DIF techniques, [8,9] we determined that the "large round bodies" as well as the cytoid and colloid bodies contained amalgamated fragments of neutrophils, keratinocytes and melanocytes, plus positive staining for CD4, CD68, fibrinogen, Complement/C3, C4, c-ANCAs and IgA. In addition, we observed a mixed immune response including $\mathrm{T}$ cells, antibodies, complement in the upper dermal inflammatory infiltrate. The immune response was seen against the BMZ, dermal vessels and eccrine glands.

Several authors have previously reported the presence of a specific $\mathrm{T}$ cell immune response in EM; that cell-mediated immunity appears to be responsible for the destruction of epithelial cells via CD8 and macrophages, and that the dermis displays an influx of CD4 positive lymphocytes in response to herpes virus infections [10]. No viral immune response was seen at least in situ in this case. Contrary to what has been described in many reports in the literature, HLA-DP, DQ, DR antigen stained weakly positive; our staining was primarily observed on inflammatory cells around the upper dermal vessels, and was negative on the vessels themselves.

Multiple medications may also trigger vasculitis without clinical EM, and ANCAs may present in other conditions [5-9]. ANCAs may be found in granulomatosis with polyangiitis, microscopic polyangiitis, Churg-Strauss syndrome and levamisole-adulterated cocaine intoxication. C-ANCAs have been described primarily to be of $\mathrm{IgG}$ isotype, directed against antigens in the cytoplasms of neutrophils. However, in previous reports we have described c-ANCAs presenting via other immunoglobulin isotypes [9]. In EM presenting with c-ANCAs and vasculitis, is also important to evaluate the patient for renal, pulmonary, joint and muscular function.

\section{CONCLUSIONS}

We found evidence of an amalgamation of multiple cells and their debris in cytoid bodies, colloid bodies and "large round bodies". These findings may represent the results of defragmentation and amalgamation of multiple cells including those involved in the immune response to EM; in addition, the findings suggest a brisk immune response. The immune response was directed inside the blisters, on epidermal cell junctions, and against dermal vessels and eccrine glands. Overall, our results may be indicative of a more complex immune response than previously documented in EM, involving cytotoxic T cells, antigen presenting cells, neutrophils and c-ANCAs.

\section{Abbreviations}

Hematoxylin and eosin (H\&E), antineutrophil cytoplasmic antibodies (ANCAs), cytoplasmic ANCA (c-ANCA), microscopic polyangiitis (MPA), antineutrophil cytoplasmic myeloperoxidase antibody (MPO-ANCA), erythema multiforme (EM), direct immunofluorescence (DIF), immunohistochemistry (IHC), basement membrane zone (BMZ).

\section{REFERENCES}

1. Huff JC, Weston WL, Tonnesen MG. Erythema multiforme: A critical review of characteristics, diagnostic criteria, and causes. J Am Acad Dermatol. 1983;8:763-75.

2. Chanda JJ, Callen JP. Erythema multiforme and the Stevens-Johnson syndrome. South Med J. 1978;71:566-70.

3. Shah SN, Chauhan GR, Manjunatha BS, Dagrus K. Drug induced erythema multiforme: Two case series with review of literature. 


\section{www.odermatol.com}

J Clin Diagn Res. 2014;8ZH01-4.

4. Abreu Velez AM, Klein AD, Howard MS. Junctional adhesion molecule overexpression in Kaposi varicelliform eruption skin lesions-as a possible herpes virus entry site. North Am J Med Sci. 2010;2:433-7.

5. Abreu Velez AM, Smith JG Jr, Howard MS. Neutrophil extracellular traps (NETS), IgD, myeloperoxidase (MPO) and antineutrophil cytoplasmic antibody (ANCA) associated vasculitides. North Am J Med Sci. 2009;1:309-13.

6. Finan MC, Schroeter AL. Cutaneous immunofluorescence study of erythema multiforme: Correlation with light microscopic patterns and etiologic agents. J Am Acad Dermatol. 1984;10:497-506.

7. Chularojanamontri L, Tuchinda P, Triwongwaranat D, Pinkaew S, Kulthanan K. Diagnostic significance of colloid body deposition in direct immunofluorescence. Indian J Dermatol Venereol Leprol. 2010;76:373-7.

8. Abreu Velez AM, Calle Isaza J, Howard MS. Immunofluorescence patterns in selected dermatoses, including blistering skin diseases utilizing multiple fluorochomes. North Am J Med Sci. 2015;7:397-402.

9. Abreu Velez AM, Brown VM, Shipp L, Smoller BR, Howard MS. N-TRAPS, C-ANCAS in a vasculitis-lupus-scleroderma-panniculitis overlap syndrome. Our Dermatol Online. 2013;4:83-6.

10. Brice SL, Stockert SS, Bunker JD, Bloomfield D, Huff JC, Norris DA, et al. The herpes-specific immune response of individuals with herpes-associated erythema multiforme compared with that of individuals with recurrent herpes labialis. Arch Dermatol Res. 1993;285:193-6.

Copyright by Ana Maria Abreu Velez, et al. This is an open access article distributed under the terms of the Creative Commons Attribution License, which permits unrestricted use, distribution, and reproduction in any medium, provided the original author and source are credited.

Source of Support: Georgia Dermatopathology Associates, Atlanta, Georgia, USA, Conflict of Interest: None declared. 